\title{
Saccadic eye movements and the perception of visual direction
}

\author{
WAYNE HERSHBERGER \\ Northern Illinois University, DeKalb, Illinois
}

\begin{abstract}
The extant research literature concerning intrasaccadic stimuli implies that if a spot of light is flashed in the dark during a saccadic eye movement, one should subsequently look for the light in one direction while professing to see it lying in another. This paper accounts for this paradox in terms of two hypothesized varieties of sensed eye orientation, one estimating actual eye orientation (efference copy) and the other corresponding to intended eye orientation (afference copy).
\end{abstract}

The purpose of this paper is to suggest a solution to a paradox. The paradox concerns saccadic eye movements and the perception of visual direction. The apparent location, or, more exactly, the visual direction of a spot of light flashed very briefly (e.g., $2 \mathrm{msec}$ ) during a saccadic eye movement executed in total darkness, is illusory. That is, the light source is perceptually mislocalized (Matin \& Pearce, 1965). Yet, if one intends to look toward that light source, a reasonably accurate refixation saccade follows in due course (Hallet \& Lightstone, 1976a, 1976b). The paradox is that one looks for the light source in one direction while professing to see it located in another (Hallet, 1976). The suggested solution to this paradox derives from a theoretical model of the oculomotor control system advanced by Robinson (1975). Robinson's closed-loop model controls eye orientation and utilizes, as do all servo systems, two separate indices of the variable being controlled: a feedback signal and a reference signal. The neural feedback signal in Robinson's model is a putative efference copy (von Holst \& Mittelstaedt, 1950), and the neural reference signal is what I have elsewhere called an afference copy (Hershberger, 1976). The thesis of the present paper is that saccadic eye movements depend upon both neural copies, whereas psychophysical judgments of visual direction depend only upon the afference copy. The former hypothesis is called the sum-of-errors hypothesis, for reasons given below. The latter hypothesis is called the afference-copy hypothesis.

This paper is organized as follows: First, a description of Robinson's model and its empirical basis is presented. Then the two hypotheses composing the present thesis are given, along with an account of how they explain the aforementioned paradox. Finally evidence relevant to each hypothesis is reviewed: For the sum-of-errors hypothesis, the evidence is direct and briefly put. The case for

Portions of this article were presented at the Annual Meeting of the American Society for Cybernetics, Philadelphia, November 1984. The author thanks Associate Editor Robert Fox and several anonymous reviewers for their constructive criticisms of preliminary drafts of this paper. The author's mailing address is: Department of Psychology, Northern Illinois University, DeKalb, IL 60115. the afference-copy hypothesis, however, is less straightforward and more complex. For one thing, it involves the consideration of two different types of supporting evidence, one newly noted by the author and one traditional. Furthermore, it also involves a reconsideration of two sets of extant data that appear to be inconsistent with the hypothesis, when in fact they are not necessarily so. These two sets of data are those that led or misled Shebilske (1976) to conclude that apparent visual direction is not determined by intended eye position, and those that led or misled Matin (1972) to conclude that perisaccadic changes of perceived visual direction occur slowly. The order of presentation is as follows: (1) Shebilske's data, (2) the evidence newly noted by the author, (3) Matin's data, and finally, (4) the evidence of a traditional variety involving judgments made by subjects whose extraocular muscles have been paralyzed.

\section{Robinson's Model}

Robinson has advanced the thesis that saccadic eye movements are but the overt manifestations of a "bangbang control system" that continuously monitors eye position and drives the eye from one intended position to another with maximal force and velocity. The system "consists of a simple negative feedback system whose forward path contains a high gain saturating amplifier with a dead zone (so it is either on or off) and an integrator"' (Robinson, 1975, p. 369). The model is in sharp contrast to Young and Stark's (1963a, 1963b) traditional sampleddata model in which eye movements, rather than eye positions, are coded and the movements themselves are preprogrammed and executed in an all-or-none, "ballistic" fashion. Strong evidence for Robinson's model came from a patient Zee and Robinson (Zee, Optican, Cook, Robinson, \& King Engel, 1976) examined who suffered spinocerebellar degeneration. Such patients make slow saccades, and this patient's saccadic velocity saturated at about $80 \% / \mathrm{sec}$. Robinson has reported that

she made saccades less than $5^{\circ}$ more or less normally but a $40^{\circ}$ saccade, for example, could take 500 or even $600 \mathrm{msec}$. Using double jumps to look for evidence of sampling [we] found that her "saccades" were not ballistic or 
preprogrammed at all and showed no evidence of sampling. When the target made its second jump, she stopped (after one reaction time) doing whatever she was doing in response to the first target jump and her eyes turned right around and started, at $80 \% \mathrm{sec}$, toward the new target position. (Robinson, 1975, p. 368)

Evidently, the ballistic appearance of normal saccades is but an illusion engendered by their brevity; that is, the fact that saccades normally proceed without interruption is perhaps merely an artifact of their latencies' exceeding their durations. Only when normal saccades are very large would they take long enough even to allow for their being interrupted, as indeed perhaps they are: Becker and Jurgens (1975) have reported data that suggest that, under certain conditions, they may be.

Even more relevant to Robinson's thesis are the experimental findings of Mays and Sparks (1980), who investigated saccadic eye movements in rhesus monkeys. They used electrical stimulation of the superior colliculus to move the eyes of a monkey just before he began a saccade to a spot of light flashed in the dark. Despite this electrode-induced perturbation, and the fact that the flashed target was no longer visible, the monkey's subsequent saccade brought his gaze to the target location, something clearly impossible had the movement been determined solely by retinal information. Apparently, the saccadic system monitors eye position as well as retinal information and points the eyes at fixation targets by commanding particular directions of gaze, namely those directions in which visible targets are seen to lie.

Robinson himself argued that the ability to point at visually fixated targets with the hand implies that eye position is known at least to some parts of the nervous system and that therefore it may very well be known to the saccadic system itself. However, "there are almost certainly differences between the sensory processes leading to perception [of target location] and those leading to saccadic eye movements" (Hallet \& Lightstone, 1976a, p. 99). For, although a fixation target flashed during a saccade "elicits a subsequent goal-directed saccade of normal amplitude and appropriate latency" (Hallet \& Lightstone, 1976b, p. 107), a stimulus flashed in darkness during a saccadic eye movement tends to be perceptually mislocalized, as the research of Matin and his associates has shown (e.g., Matin, 1972, 1982; Matin \& Pearce, 1965). Also, a saccade made during a change of fixation to a target at a different distance is directed toward the target's actual rather then perceived location (Ono \& Nakamizo, 1977).

Hence, if the saccadic system does monitor eye position, as Robinson suggests, it does not appear to be the same measure of eye position that is responsible for the apparent location of visual discriminanda. Apparently there are two different measures of eye position monitored by the nervous system, one involved in the determination of saccadic eye movements and another involved in the visual perception of an object's location in space. Fortunately, Robinson's hypothesis provides parsimoni- ously for just such a possibility. In any closed-loop control system, such as Robinson is hypothesizing, the value of a controlled variable is driven into correspondence with the value of a reference variable by means of negative feedback. In Robinson's case, the latter is the eye's intended position or orientation and the former is the eye's controlled position or orientation. Since both these variables represent eye orientation and both are manifestly neural, they provide two potential indices of eye orientation that the nervous system may monitor.

Robinson assumes that the eye's controlled position is monitored as a form of sensed efference, "efference copy" (von Holst \& Mittelstaedt, 1950), or "corollary discharge"' (Sperry, 1950). That is, Robinson believes the saccadic control system senses eye orientation by monitoring its own neuromuscular commands. This contrasts with Sherrington's (1918) suggestion that the stretch receptors in the extraocular muscles sense eye orientation. However, it matters little to Robinson's closed-loop model just how eye orientation is sensed; his fundamental thesis is that eye orientation, however it is sensed, is compared with and driven into correspondence with an intended orientation by means of negative feedback. It is this notion of an intended eye orientation that distinguishes Robinson's saccadic control model. It is to be noted that this intended eye orientation is but a particular eye orientation that the control system intends to sense. It is a neural copy of the sensation intended. Elsewhere, Hershberger (1976) has called such copies of intended sensation "afference copies"' in order to contrast them with von Holst and Mittelstaedt's (1950) concept of "efference copies." Robinson's model of the saccadic control system incorporates both types of copies. In Robinson's model, the intended eye orientation is an afference copy, the sensed eye orientation is an efference copy, and the control loop drives the efference copy into correspondence with the afference copy by means of negative feedback.

It is important to note that the expression afference copy employs the term copy in its archaic sense to mean something that is to be imitated (e.g., a prototype), whereas von Holst and Mittelstaedt's expression efference copy employs the term copy to mean that which is an imitation.

\section{The Present Thesis: Accounting for the Paradox}

The existence of these two different neural copies, each an index of eye orientation (one intended and one sensed), provides for a possible accounting of the paradoxical fact that a spot of light flashed during a saccade is perceptually mislocalized and yet capable of eliciting a subsequent refixation saccade of normal accuracy.

The afference-copy hypothesis. The explanation advanced here, as a corollary hypothesis to Robinson's thesis, is that the perceived direction of gaze, which determines the perceived location of visible objects, corresponds not to the eye's sensed orientation but to its intended orientation: in other words, not to its efference copy but to its afference copy. Call this speculation the afference-copy hypothesis. According to this afference- 
copy hypothesis, a spot of light flashed onto the fovea (i.e., line of sight) of a moving eye should appear to lie in a direction corresponding to the movement's intended goal rather than to the eye's actual orientation at the time of the flash. That is, the flash should be perceptually mislocalized.

The sum-of-errors hypothesis. Implicit in the afference-copy hypothesis is the assumption that this neural variable, the afference copy, may be altered in value incrementally from one intended orientation to another, as, for example, in the fixation of successive flashing lights. And it is further speculated that in such cases the angular magnitude of such incremental alterations of the afference copy is simply the sum of two angular error signals: (1) the retinal eccentricity of a stimulus flash, and (2) the oculomotor error signal at the time of the flash. Call this speculation, illustrated in Figure 1, the sum-oferrors hypothesis. According to the sum-of-errors hypothesis, if a spot of light flashed upon the fovea (i.e., the line of sight) of a moving eye is taken as a target to be fixated, the oculomotor error signal existing at the time of the flash serves to increment the afference copy so that

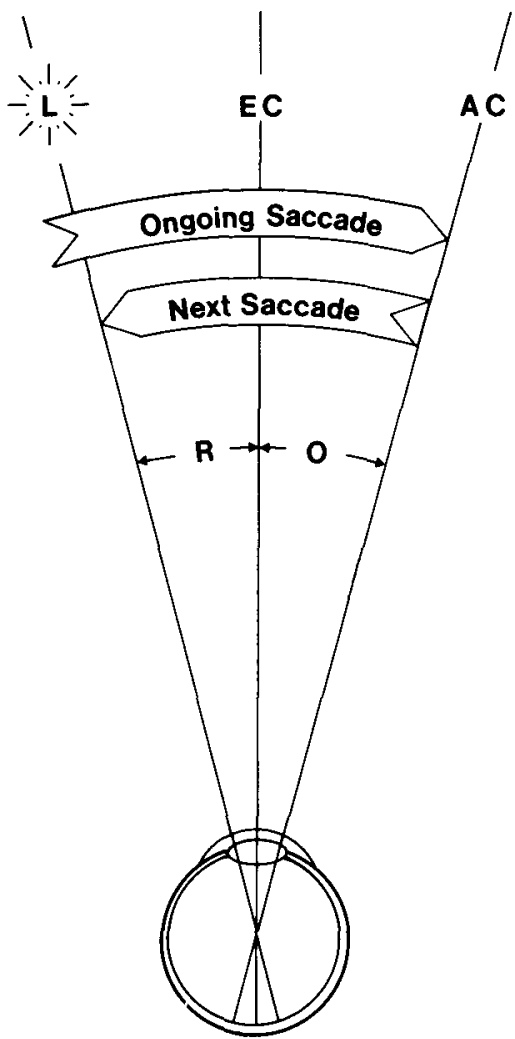

Figure 1. A point light source, $L$, is flashed briefly during a clockwise saccade to an intended orientation, $\mathrm{AC}$, represented by an afference copy. The actual eye orientation at the time of the flash, EC, is sensed by means of an efierence copy. According to the sumof-errors hypothesis, the oculomotor control system calculates the size of the next saccade by summing $R$, the retinal eccentricity of the image, with $O$, the oculomotor error signal (AC-EC) at the time of the flash. a subsequent successful refixation saccade follows a reaction time later. Thus, together, these two hypotheses, the afference-copy hypothesis and the sum-of-errors hypothesis, account successfully for the aforementioned paradoxical findings of Matin et al., on the one hand, and Hallet and Lightstone, on the other.

\section{Evidence for the Sum-of-Errors Hypothesis}

The extant research literature provides evidence that supports each of these hypotheses. On behalf of the sumof-errors hypothesis, there is the research of Goldberg and Bruce, who have been studying frontal-eye-field neurons in monkeys (Bruce \& Goldberg, 1981; Goldberg \& Bruce, 1981). They have reported finding three types of cells in the frontal eye fields, which appear to correspond exactly to the two hypothesized error signals and their sum. In Goldberg's own words, "In the frontal eye fields ... there are cells that discharge according to the retinal location of a stimulus, according to the direction and amplitude of the most recent eye movement, and according to the metrics of the next visually guided saccade (Bruce \& Goldberg, 1981). In a two-jump experiment, cells discharge not according to the retinotopic target location or the spatial target location, but rather according to the eye movement needed to acquire the target. There is no static map of the world, just a map of saccades. A given retinal stimulus can evoke any saccade, given the proper antecedent eye movement. The spatial map is only a virtual map, linked to the motor output by the recent eye movement" (Goldberg, 1983, p. 21). In other words, for saccades mediated by frontal-eye-field neurons, the two hypothesized error signals and their sum are sufficient not only in principle but in fact!

\section{Evidence for the Afference-Copy Hypothesis}

Shebilske's data. Turning to the evidence for the afference-copy hypothesis, it is appropriate to begin with the work of Shebilske (1976), who first advanced and subsequently abandoned such a notion. Shebilske was investigating the nature of corrective secondary saccades that follow dysmetric primary saccades elicited by spots of light flashed in the dark. Typically, the dysmetric primary saccade is hypometric; that is, the eye movement falls short of the now invisible target and a short-latency secondary saccade corrects for this shortcoming, all without benefit of a visual stimulus. Shebilske assumes that the eccentric orientation of the target flash is fully and accurately determined prior to the appearance of the dysmetric primary saccade, and that the corrective secondary eye movement that subsequently occurs in the dark results from the error signal's representing the difference between the accurately preintended terminal eye orientation and the erroneous intermediate eye position at the end of the dysmetric primary saccade. Hence, Shebilske accepts the notion championed by Robinson that the saccadic system controls eye position and does so by means of negative feedback, a view Shebilske credits to Weber and Daroff 
(1972). Furthermore, he accepts the possibility that actual eye position may be sensed centrally by means of an efference copy, just as Robinson hypothesizes, a view Shebilske credits to Johnson (1963). However, Shebilske discounts the corollary possibility that perceived eye orientation corresponds to intended rather than actual eye orientation. That is to say, he rejects the afference-copy hypothesis. He based the latter decision upon the results of a straightforward experimental test in which a subject was to judge the location of an intersaccadic discriminandum, a light flashed briefly during the latency of a secondary saccade. Shebilske located this light flash at one of two positions, either at the location of the fixation target or on the subject's line of sight as it came to rest at the end of the dysmetric primary saccade. Were the afferencecopy hypothesis true, it would appear to follow that when the test flash was actually located on the subject's line of sight he should see it as being coincident with the terminal fixation target. Furthermore, he should see a test light located at the terminal fixation target as being located elsewhere. The opposite results obtained. Shebilske (1976) therefore concluded that apparent visual direction was "determined by an extraretinal signal that encodes actual rather then intended eye position" (p. 628).

Although Shebilske's empirical argument appears sound, his conclusion is not fully warranted, for the argument is based upon a questionable assumption, namely that both the primary and secondary saccades result from a single preintended terminal eye position. In contrast, Robinson's model of the saccadic system requires a different intended eye position for each saccade, primary and secondary alike. Hence, the afference-copy hypothesis remains tenable, at least as a corollary to Robinson's closedloop model, inasmuch as the afference-copy hypothesis predicts not that intersaccadic discriminanda should be perceptually mislocalized, but rather that intrasaccadic discriminanda should be perceptually mislocalized. More specifically, during a saccade, a foveal flash should appear to be located at the movement's intended goal or destination. The critical question is, does it? Where does a spot of light appear to lie when it is flashed upon the fovea during a saccadic eye movement?

Some relevant and suggestive results have recently been reported by O'Regan (1984). Using a photoelectric eyetracking system to monitor eye position and a cathode ray tube to present brief spots of light at predetermined locations, O'Regan programmed an on-line computer to stimulate the fovea of his subject's retina at various times either before, during, or after a saccadic eye movement. Following each stimulus presentation, the subject moved a cursor across the screen of the cathode ray tube to the point where the flash had appeared to originate.

O'Regan found that one of his 3 subjects always located the foveal flash either at the fovea's departure point or at its arrival point and never near its veridical position in between. Furthermore, and more directly to the present point, the foveal flashes that occurred during a saccade were virtually always located at the arrival point, just as the afference-copy hypothesis predicts. O'Regan's second subject performed similarly, although his settings exhibited more variability. The data from his third, and final, subject were very noisy and difficult to interpret. Although the individual differences among these 3 subjects is disconcerting, a fundamental pattern is nonetheless apparent: the perceived direction of gaze appears to correspond not to the controlled orientation of the eye as represented by an efference copy but to the intended orientation of the eye as represented by an afference copy. Thus, during a saccadic eye movement, while the direction of gaze changes continuously from one orientation to another, the perceived direction of gaze appears to change discontinuously from one intended orientation directly to another without ever assuming any of the possible intervening orientations.

Because O'Regan's subjects were tested under normal lighting conditions, with the initial and terminal fixation targets (luminous triangles) visible throughout each trial, the retinal image of the terminal fixation target eventually became superimposed upon the retinal remanence of the test flash (the letter I) imaged upon the fovea during the saccade. Hence, seeing the two, the I and the terminal triangle, as occupying the same relative position may merely have reflected the fact that they both shared the same retinal locus at trial's end. Thus, O'Regan's intriguing results, although fully consistent with the afferencecopy hypothesis, may not be regarded as definitive evidence that the extraretinal signal mediating perceived direction of gaze changes discontinuously with each intended refixation (cf. Matin, 1976, Figure 5).

However, there are two types of experimental observation that do appear to provide such evidence--one traditional and one new. The new variety involves psychophysical judgments of a type each reader may make for himself/herself; the traditional variety involves judgments made by subjects whose extraocular muscles have been paralyzed. Consider first the new variety.

New evidence. If one fixates alternately to the left and right of a rapidly blinking light viewed in the dark, one will see a spatially extended series of phantom lights blinking on and off sequentially in a direction opposite to that of the saccade. ${ }^{2}$ The direction of this motion appears merely to reflect the direction of the retina's motion across the blinking image. What is remarkable is that the perceived shift in the direction of regard is reflected only in what appears to be a discrete displacement of the entire array of phantom lights in the direction of the eye movement. For instance, if the arrow in Figure 2 represents a single saccadic eye movement and the asterisk represents a single flashing light, the bracketed array represents the phenomenal appearance. One sees, fixed in space, a horizontal array of lights that blink on and off in sequence, giving an impression of apparent motion, or phi. The phantom array does not itself appear to move; however, neither is it centered upon the light. Rather, in the case illustrated above, the array is displaced to the right, with its left end appearing to be located in the light's presac- 


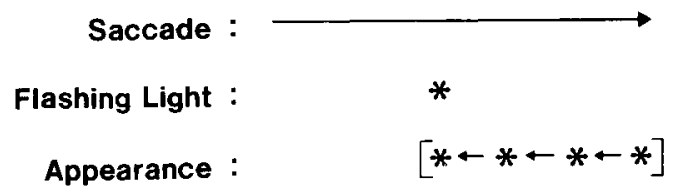

Figure 2. If you shift your gaze saccadically from the left to the right of a point light source in a darkened room, blinking on and off at $120 \mathrm{~Hz}$, you will see phi movement to the left within a phantom array that is displaced to the right.

cadic direction. Because the flash seen on the right end of the phantom array is painted onto the retina first, that is, before any of the other flashes seen in the array, and because the gaze continues to shift to the right as the remaining flashes in the array are being painted onto the retina, the retinal locus of the remanence of the first flash moves through a substantial visual angle equally as large as the phantom array itself. If the local sign (perceived visual direction of that retinal locus) shifted continuously and isometrically with the eye movement, then the first flash seen should appear to move rightward in the direction of the changing gaze by an angular amount equal to that subtended by the phantom array. But it does not appear to move to the right at all. Rather, it appears to be displaced, or placed, to the right by the observed amount all at once without having moved through the intermediate locations. Neither are any of the other flashes in the array seen to move to the right. They are seen to be placed to the right but not to be moving to the right. This is true in spite of the fact that the saccade does not itself preclude motion perception: phi to the left is clearly visible within the phantom array. This implies that changes of perceived direction of regard are either entirely presaccadic or very abrupt-or both.

This is not to say that the changes of perceived direction of regard that accompany normal saccadic eye movements never engender perceptual errors of visual direc-

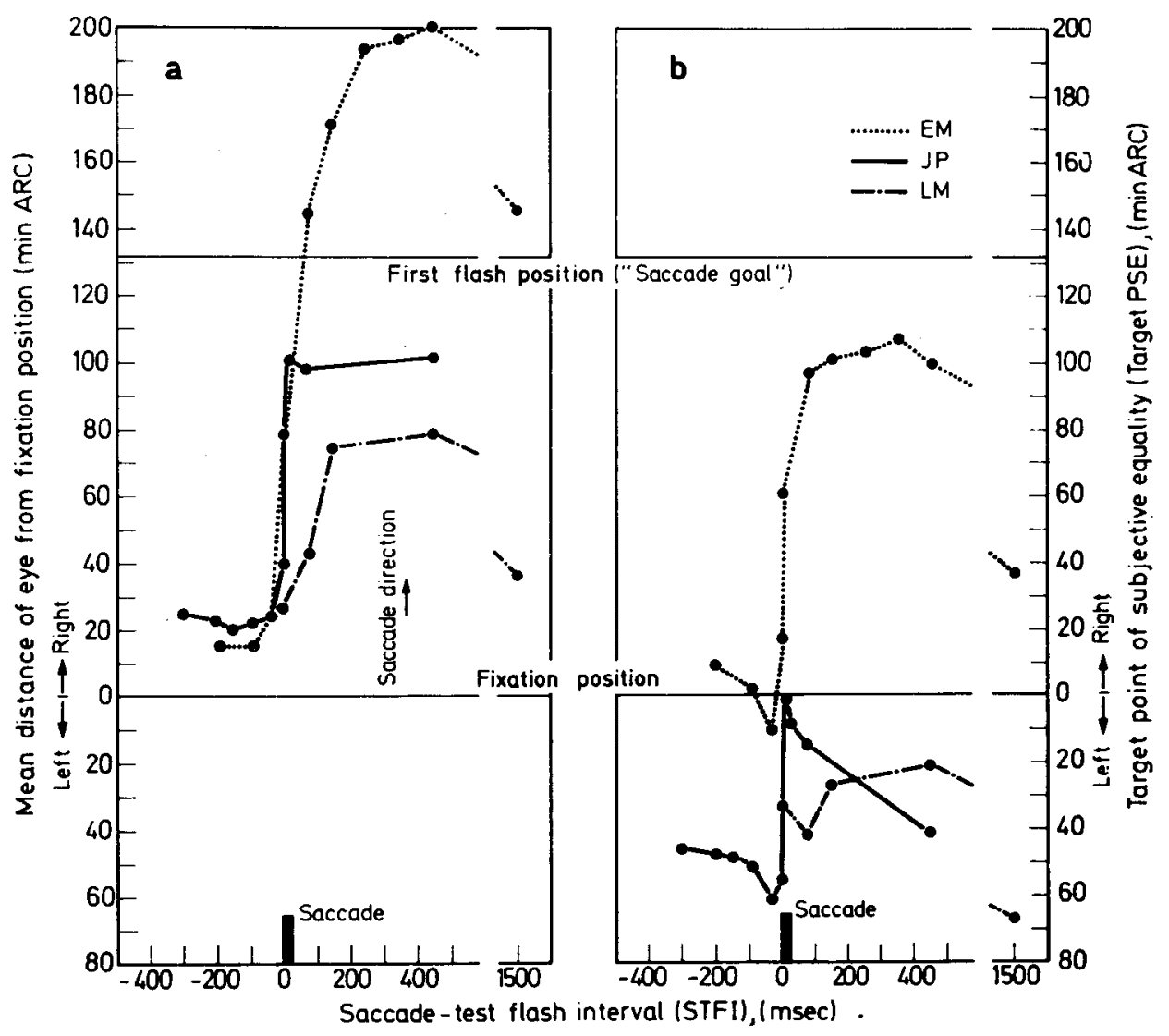

Figure 3. “(a) Mean eye position at times before, during, and after saccade when test fashes were presented. (b) Points of subjective equality at the stimulus array determined from the subject's report of the borizontal visual direction of the test flash relative to previously viewed fixation target. Negative values of STFI refer to trials in which the test flash was presented before the saccade. It was not posible to reproduce on this graph all of the points during the saccade; only one or two are shown here. Presaccadic data for E.M. are for leftgoing saccades; all the rest are for rightgoing saccades, as shown. Graph compiled from data in Matin, Matin, \& Pearce, 1969; Matin, Matin, \& Pola, 1970; also in preparation." (Figure and quoted caption from "Eye Movements and Perceived Visual Direction" by L. Matin, 1972. In D. Jameson \& L. Hurvich [Eds.], Handbook of Sensory Physiology, Vol. VII/4 [p. 347]. Heidelberg: Springer-Verlag. Copyright 1972 by Springer-Verlag. Reprinted by permission of the publisher and the author.) 
tion, but only that such changes and whatever errors they do engender manifest themselves as visible displacements rather than visible motions. The apparent motion visible in the phantom array reflects an error of visual direction that is being reduced by the saccade. The error itself, as it appears initially, is already fullblown, manifesting itself as a discrete displacement.

Matin's data. Researchers using Fechner's frequency method (see Woodworth \& Schlosberg, 1954) to determine the location of a single perisaccadic test flash that will appear to lie in the same direction as a remembered prior fixation target, no longer visible, have found constant errors of visual direction to occur throughout an extended interval of time, ranging from several hundred milliseconds before the saccade to many hundreds of milliseconds after the saccade (Matin, 1972, 1982). For instance, for a small saccade of about $2^{\circ}$, the range of errors for 1 subject (Subject E.M., Figures 3 and 4) was found to extend from about $200 \mathrm{msec}$ before the saccade to $1,500 \mathrm{msec}$ after the saccade. (Figures 3 and 4 are reprints of Figures 9 and 10 from Matin, 1972: Figure 3b shows the positions of perisaccadic test flashes presented at various saccade-test-flash intervals, STFI, which subjects judged as lying in the same direction as a remem-

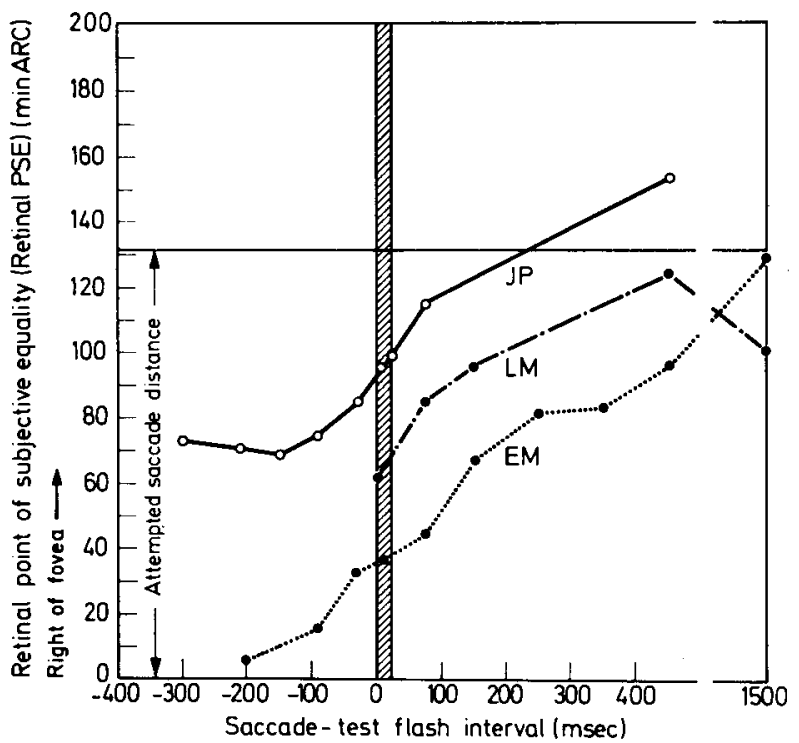

Figure 4. "Points of subjective equality for the fixation target measured as horizontal distances at the retina. These are plotted as a function of time relative to saccade onset. The data are from the same experiments as the data in Figure 3. Although there is a close correspondence between the retinal PSEs and the difference between mean eye position and target PSEs at corresponding values of STFI, it is not exact; this is mainly a result of the fact that distributions of eye positions at fixed values of STFI were frequently skewed. Each retinal PSE was calculated directly from this distribution of retinal distances and psychophysical responses over trials at a given value of STTT." (Figure and quoted caption from "Eye Movements and Perceived Visual Direction" by L. Matin, 1972. In D. Jameson \& L. Hurvich [Eds.], Handbook of Sensory Physiology, Vol. VII/4 [p. 348]. Heidelberg: Springer-Verlag. Copyright 1972 by SpringerVerlag. Reprinted by permission of the publisher and the author.) bered presaccadic fixation target located at the origin; these are called target PSEs. Assuming that memory for the locus of the fixation target is accurate, the target PSEs reflect constant errors of perceived visual direction of the test flash. The retinal PSEs shown in Figure 4 were calculated by combining mean recorded eye position with target PSE at corresponding STFIs. Mean eye position over all trials is shown in Figure 3a.) It is tempting to suppose that the constant errors detected in this $1.7-\mathrm{sec}$ interval reflect the time course of a sluggish extraretinal signal mediating perceived direction of regard. However, reasonable as it may seem, such a supposition is but a supposition. Rather than reflecting a sluggish extraretinal signal that takes nearly $2 \mathrm{sec}$ to undergo a change of $2^{\circ}$, the protracted interval of constant errors may reflect brisk changes of perceived visual direction that occur with a latency that varies substantially from trial to trial. For instance, suppose, for the sake of argument, that the shift in retinal local signs that attends a saccade occurs in a discrete, stepwise fashion, and that the latency, but not size, of this step varies from trial to trial. Successive trials of repeated stimulation of the same retinal locus at the same relative time (e.g., $20 \mathrm{msec}$ prior to eye movement) will yield a bimodal distribution of apparent visual direction, one mode comprising the effects of the trials on which the stimulus precedes the shift and the other comprising the effects of the trials on which the stimulus follows the shift. The central tendency of the distribution as a whole, customarily taken to represent the true visual direction or local sign of the retinal signal, may be observed to depend heavily upon the relative frequencies of the two types of trials, which, in turn, depend heavily upon when during the perisaccadic interval the stimulus is presented. In general, the later the stimulus occurs in the interval, the more frequent the postshift trials are likely to be and, hence, the greater the apparent shift in local signs, even though the actual shifts are all of the same magnitude whenever they occur.

The afference-copy hypothesis states that discrete changes of intended eye orientation are accompanied by corresponding changes in perceived visual direction, but these abrupt changes need not be synchronous. Synchronicity is only the simple case. It would not be dysfunctional if the discrete change in perceived visual direction were to follow the change of intended eye orientation by a variable delay that tended, on the average, to synchronize the perceived change with the midpoint of the saccade. Conceivably, the perceived change could occasionally follow the saccadic motion altogether. A variable latency such as this could reasonably account for some of the gradual shifts in local sign apparent in Figure 4 (Matin's Figure 10), at least, the presaccadic data of Subjects L.M. and J.P.

However, it is implausible that latency variability could account for such belated constant errors of visual direction as those evidenced by the psychophysical judgments of Subject E.M. Skavenski (1976; Skavenski \& Stein- 
man, 1970) has suggested that these belated constant errors reflect drift in the subject's memory for the location of the presaccadic fixation target whose position is being judged, and do not reflect errors of perceived visual direction in the first place (cf. Matin, Pearce, Matin, \& Kibler, 1966). Whatever the merits of Skavenski's idea, one cannot help but be impressed by significant differences between Subject E.M. and the other two subjects (L.M. and J.P.), whose data are summarized in Figures 3 and 4.

Figure 4 shows, for each subject separately, the retinal eccentricity of perisaccadic test flashes, plotted as a function of time from saccadic onset, which appear to originate from the same place as a presaccadic fixation target. Assuming that memory for the location of the prior fixation target is accurate, these retinal points of subjective equality (PSEs) reflect the subjects' shifts in retinal local signs. Each subject's shift begins before the saccade begins and grows over time. The afference-copy hypothesis predicts that the size of this shift should come to equal the size of the saccade no later than the occurrence of the saccade itself. ${ }^{3}$ To assist the reader in determining when the shift has reached this level, Matin drew a single horizontal line across the figure at 131' of arc and labeled it "attempted saccade distance." This single line, however, does not suffice. What is needed are three such lines, a different line for each subject, because these 3 subjects differed dramatically in the size of their typical saccade, despite the fact that each saccade was invariably triggered by an eccentric point light flash located 131' of arc from the subject's presaccadic fixation point.

Although J.P.'s and L.M.'s saccades typically undershot the locus of the trigger flash, E.M.'s saccades "typically (but not invariably) overshot the position of the first [i.e., trigger] flash ... by as much as $.5 \mathrm{deg}$ to $1 \mathrm{deg}$ '” (Matin, Matin, \& Pearce, 1969, p. 71). Although the size of each subject's typical saccade was not reported, one gets an impression of the size from an inspection of Figure 3a, which shows the mean eye position of each subject plotted as a function of time from saccadic onset. ${ }^{4}$ The three curves reach their respective asymptotes at about 80' (L.M.), 100' (J.P.), and 200' (E.M.) of arc from the prior fixation point. Now, if one draws into Figure 4 three horizontal lines corresponding to these three asymptotes, one finds a picture that is altogether different from that framed by the single reference line drawn at $131^{\prime}$ of arc.

First, one is able to see that the function representing E.M.'s shift in retinal local signs never comes even close to (not within $60^{\prime}$ of arc of) reaching her saccadic asymptote. This shortfall is so great that one must doubt whether E.M.'s psychophysical function even represents shifting retinal local signs in the first place. It is more plausible that, as Skavenski suggests, the function reflects a drift in memory for the location of the presaccadic fixation target.

In stark contrast to E.M.'s data, J.P.'s shift of retinal PSEs reaches a level equal to his saccadic asymptote and does so during the course of the saccade itself, just as the afference-copy hypothesis predicts. At this time, J.P.'s errors of perceived visual direction should be zero (providing his memory for the location of the presaccadic fixation point is accurate), and a test flash whose location he judges to be subjectively equal to that of the prior fixation point would actually have to originate from that location. An examination of Figure 3b, showing such target PSEs for all 3 subjects, reveals that the constant error of J.P.'s target PSE was indeed zero at this time. This constant error subsequently grew from zero at saccade's end to about $40^{\prime}$ of arc $.5 \mathrm{sec}$ later, but, as Skavenski suggests, this spontaneous change for the worse is better attributed to drift in memory than to any putative imprecision of extraretinal signals per se.

L.M.'s data are similar to J.P.'s in that L.M.'s shift in retinal PSEs reaches the level of his saccadic asymptote and does so near the very end of the saccade. There is an important difference, however. Although the constant error of J.P.'s target PSEs momentarily drops to zero just as his retinal PSE comes to equal his saccadic asymptote, the constant error of L.M.'s target PSEs never falls below $20^{\prime}$ of arc. Hence, one is probably not warranted in assuming that L.M.'s shifting retinal PSEs are entirely free of errors of memory for the location of the fixation target and, hence, reflect only shifting retinal local signs. This being the case, it is not possible to identify the precise moment when the size of the saccade and the size of its attendant shift in retinal local signs become isometric. Hence, L.M.'s data, although promising, are too imprecise to be considered as evidence for (or against) the hypothesis.

What the above analysis suggests is that at least some of the psychophysical functions plotted in Figure 4 are composites of at least two processes: shifts in retinal local signs and drifts in memory, or the like. The former is visible as a quadratic component in the presaccadic portion of J.P.'s data. The latter is visible as a linear component in the postsaccadic portion of J.P.'s data. Since there is little evidence that E.M.'s data reflect shifts of local signs, it is not surprising to find that her psychophysical function is rectilinear throughout and virtually parallel to J.P.'s linear component.

By taking the individual differences among these 3 subjects seriously into account, it becomes apparent that data from one (J.P.) are precisely consistent with the afferencecopy hypothesis, data from a second are promising but too imprecise to judge, and the idiosyncratic data from the third are merely equivocal. Thus, these classic data provide much the same level of support for the hypothesis as does O'Regan's data described above. That is, some of these data provide clear evidence for the hypothesis; none are clear evidence against it.

Perhaps the most telling evidence to come from Matin's laboratory has been that reported by Pola (1976), who used McLaughlin's (1967) technique for conditioning parametric adjustments of saccadic eye movements. McLaughlin had found it possible to condition a reduction in the size of saccades used to shift fixation from tar- 
get $A$ to target $B$ by extinguishing $B$ during each saccade and replacing it with a surrogate target $B^{\prime}$ situated closer to $\mathrm{A}$.

Using McLaughlin's conditioning technique and Matin's (1972) psychophysical procedures for determining the retinal PSE of target A during saccades from A to B/B', Pola found that, whereas the size of a normal 8-deg saccade could be conditionally reduced to, say, $5 \mathrm{deg}$, the accompanying shift of target A's retinal PSE still resembled that which normally accompanies an 8-deg saccade. (Hershberger \& Misceo, 1983, p. 395)

In general, for any observed saccade of a given size, the size of the corresponding shift of visual direction varies with the size of the saccade the subject is attempting to make (cf. McLaughlin, Kelly, Anderson, \& Wenz, 1968; Miller, 1980). Pola's finding is precisely consistent with the afference-copy hypothesis, which predicts that perceived visual direction should correspond to intended and not merely actual eye orientation. Of course, Pola's finding appears to be consistent with any version of Helmholtz's hoary hypothesis (see Hershberger \& Misceo, 1983). However, turning attention to the second type of experimental observation alluded to above (vision with extraocular paralysis), we find evidence that appears consistent in detail only with the afference-copy version of the Helmholtzian hypothesis.

Traditional evidence. If a person's eyes were to be totally immobilized mechanically, pharmacologically, or by virtue of clinical pathology, each intended change of gaze should alter his or her perceived direction of gaze in a stepwise fashion so that a static visual scene imaged upon his or her immobile retina would appear to be egocentrically displaced. The scene need not appear to move as such, at least not any more than it does when the eye moves normally, which is to say not at all. It need only appear to be egocentrically displaced in the sense that any thought of reaching out and touching visible objects lying on the line of sight would seem to require an arm extension in a direction consistent with the intended direction of gaze, and that if the person were indeed able to extend his or her arm in this direction his or her reaching out would exhibit "past pointing." Of course, past pointing at eccentric visual targets is not an inevitable consequence of extraocular paralysis. Just as it is possible to point one's hand and arm accurately at a retinally eccentric target viewed with an immobile normal eye staring straight ahead, so it would be possible to do so with an immobile paralyzed eye staring straight ahead. Past pointing is to be expected only to the degree to which the direction in which one is pointing the hand is specified by erroneous extraretinal information.

Stevens et al. (1976) have reported experimental findings that are consistent in detail with these implications of the afference copy hypothesis. They examined the effects of both partial and total ocular immobilization produced either by means of a local anesthetic (procaine) injected into the extraocular muscle cone or by means of a neuromuscular blocking agent (curare/succinylcholine) administered systemically. They found that partial paralysis produced by either means was associated with past pointing and visual displacement punctuated by a noticeable jumping of the visual scene.

This was described as a sensation of displacement rather than actual movement. "The world did not move.... It was not as if you had taken the stimulus and moved it across the screen.... When I moved my eyes up [the stimulus] disappeared and then popped up again in another place."

The displacement was preceded either by a very rapid jump or a blanking out of the visual input during the saccades. [Subjects] ACR and RCE felt that it was a jerk or jump and JKS felt that it was sometimes a jerk and sometimes a blanking out. This perception of blanking out or rapid jerk [is what is meant by the term] jumping. (Stevens et al., 1976, p. 95)

These perceptual effects of partial extraocular paralysis, as reported by Stevens et al., appear consistent with clinical observations (e.g., Cogan, 1956; Jackson \& Paton, 1909; Helmholtz, 1867/1962) and experimental findings of others (Brindley \& Merton, 1960; Kornmueller, 1931; Siebeck, 1953, 1954; West, 1932).

Stevens et al. subjected only one individual to total extraocular paralysis, but this one subject, J.K.S., was administered each type of injection, local or systemic, on three separate occasions. After the first systemic injection of succinylcholine,

JKS reported no movement or displacement during attempted saccades. "I tried to move my eyes as hard as I possibly could and nothing happened, the world was just there... I I simply could not move my eyes." [After the second systemic injection, J.K.S. again] ... reported that he was very much aware that his eyes were paralyzed. "I know I did not move my eyes. I was trying very hard." However, unlike the first total paralysis experiment, "When I looked to the right I felt that if I had to touch anything ... I would have to reach over to the right.' JKS felt that his perceptions were much the same as seen during the low dose experiments, but this displacement was not punctuated by jumping. (Stevens et al., 1976, p. 95)

After the third systemic injection, J.K.S. reported the same effects he had reported after the second.

When total extraocular paralysis was achieved [by means of a retrobulbar injection of procaine] JKS reported the same perception of displacement without noticeable jumping, as seen in the succinylcholine experiments.... Past pointing during the total block was very strong. During one study JKS attempted to touch an object in the periphery and overshot by 20 in. (Stevens et al., 1976, p. 96)

These reported effects of total extraocular paralysis, both the past pointing and the displacement without jumping are precisely what is to be expected from the afferencecopy hypothesis. The displacement without jumping appears particularly significant, for the afference-copy hypothesis is the only version of Helmholtz's $(1867 / 1962)$ "effort of will," which implies that the displacements accompanying saccadic intentions should be discrete. Sperry's (1950) notion of a corollary discharge of effer- 
ence and von Holst and Mittelstaedt's (1950) efferencecopy hypothesis both imply that such displacements should appear to be continuous, at least as continuous as a conventional saccade. So, to the degree to which these findings of Stevens et al. support Helmholtz's thesis in general, they support the afference-copy hypothesis in particular.

Although the findings of Stevens et al. are consistent with the afference-copy hypothesis, they are inconsistent with the findings of Siebeck (1953, 1954; Siebeck \& Frey, 1953) and Brindley, Goodwin, Kulikowski, and Leighton (1976), who failed to note any displacement or pastpointing effects from total extraocular paralysis. Perhaps the subjects in these two studies merely failed to note the displacement, as had Stevens (J.K.S.) himself in his first experimental session; and, past pointing, as noted above, is not inevitably a predicted effect. Perhaps this accounts for the disparate findings. Indeed, there is good reason to believe that such is the case in fact. Matin et al. (1982) have shown that a well-illuminated visual field serves to mask some of the perceptual effects of partial paralysis, and suggest that it may similarly influence the effects of full paralysis as well. For instance, although a single stationary spot of light in the dark appears to be displaced whenever a partially paralyzed subject (systemic curare) moves his eyes, that same subject fails to note any change in the direction of a target judged to be straight ahead when he looks about a well-illuminated room. Matin et al. therefore recommend that the total-paralysis experiments be repeated in darkness, an implication being that such effects as those noted by Stevens et al. may thereby be fully replicated. Assuming that such expectations are, in fact, warranted, the evidence for the afference-copy hypothesis appears compelling.

Incidental evidence. Finally, it is to be noted that the phenomena that contribute to saccadic suppression provide what might be termed coincidental evidence for the afference-copy hypothesis. Saccadic suppression, the suppression of retinal signals and their detection during saccadic eye movements, tends to render retinal input spatially discontinuous. This being the case, there is no need for perceived direction of gaze to correspond to anything but discrete eye orientations. Indeed, it would appear to be dysfunctional were it otherwise. Conversely, the oculomotor control system, which is able to execute saccadic eye movements to targets flashed during a saccade, must itself be able to sense or estimate the continuously changing eye orientations during a saccade and ought, correspondingly, to be immune to the effects of saccadic suppression, just as Hallet and Lightstone (1976a, 1976b) have found.

\section{Conclusion}

In conclusion, it appears safe to say that a notion first advanced and subsequently rejected by Shebilske (1976) remains yet a very viable hypothesis. That notion is that the extraretinal signal mediating perceived direction of gaze corresponds to intended (afference copy) rather than controlled (efference copy) eye orientation. Furthermore, assuming, as does Robinson (1975), that saccadic eye movements depend upon both types of neural copies, it is possible to account for the paradoxical fact that a spot of light flashed in the dark during a saccade may at once be perceptually mislocalized and yet elicit a subsequent goal-directed saccade.

\section{REFERENCES}

BECKER, W., \& JuRGENS, R. (1975). Saccadic reactions to double-step stimuli: Evidence for model feedback and continuous information uptake. In G. Lennerstrand, P. Bach-y-Rita, C. C. Collins, A. Jampolsky, \& A. B. Scott (Eds.), Basic mechanisms of ocular motility and their clinical implications. New York: Pergamon Press.

Brindley, G., GoodwIN, G., KulJKowskI, J., \& Leighton, D. (1976). Stability of vision with a paralyzed eye. Journal of Physiology (London), 258, 65P-66P.

Brindley, G. S., \& Merton, P. A. (1960). The absence of position sense in the human eye. Joumal of Physiology (London), 153, 127-130.

Bruce, C. J., \& Goldberg, M. E. (1981). Frontal eye fields in the monkey: Classification of neurons discharging before saccades. Neuroscience Abstracts, 7, 131.

CoGAN, D. G. (1956). Neurology of the ocular muscles. Springfield, IL: Thomas.

Goldberg, M. E. (1983). Iconoclasm avoided: What the single neuron tells the psychologist about the icon. Behavioral \& Brain Sciences, 6, 20-21.

Goldberg, M. E., Bruce, C. J. (1981). Frontal eye fields in the monkey: Eye movements remap the effective coordinates of visual stimuli. Neuroscience Abstracts, 7, 131.

Ha Llet, P. E. (1976). Saccades to flashes. In R. A. Monty \& J. W. Senders (Eds.), Eye movements and psychological processes. New York: Erlbaum.

Hallet, P. E., \& Lightstone, A. D. (1976a). Saccadic eye movements towards stimuli triggered by prior saccades. Vision Research, 16, 99-106.

Hallet, P. E., \& Lightstone, A. D. (1976b). Saccadic eye movements to flashed targets. Vision Research, 16, 107-114.

HeLmholtz, H. voN. (1962). Treatise on physiological optics. (J. P. C. Southall, Ed. and Trans., Vol. 3). New York: Dover. (Original work published 1867)

Hershberger, W. (1976). Afference copy, the closed-loop analogue of von Holst's efference copy. Cybermetics Forum, 8, 97-102.

Hershberger, W., Misceo, G. (1983). A conditioned weight illusion: Reafference learning without a correlation store. Perception \& Psychophysics, 33, 391-398.

JACKSON, J. H., \& PATON, L. (1909). On some abnormalities of ocular movements. Lancet, 176, 900-905.

JoHnson, L. E., JR. (1963). Human eye tracking of aperiodic target functions (37-B-63-8). Cleveland, OH: Systems Research Center, Case Institute of Technology.

KORNMUELuER, A.E. (1931). Eine experimentelle Anasteise der ausseren Augenmuscheln am Menschen und ihre Auswirkungen. Journal fur Psychologie und Neurologie, 41, 354-366.

Matin, L. (1972). Eye movements and perceived visual direction. In D. Jameson \& L. Hurvich (Eds.), Handbook of sensory physiology (Vol. 7). Heidelberg: Springer.

Matin, L. (1976). Saccades and extraretinal signal for visual direction. In A. Monty \& J. W. Senders (Eds.), Eye movements and psychological processes. New York: Erlbaum.

Matin, L. (1982). Visual localization and eye movements. In W. A. Wagenaar, A. H. Wertheim, \& H. W. Leibowitz (Eds.), Symposium on the study of motion perception. New York: Plenum.

Matin, L., Matin, E., \& Pearce, D. G. (1969). Visual perception of direction when voluntary saccades occur: I. Relation of visual direction of a fixation target extinguished before a saccade to a flash presented during the saccade. Perception \& Psychophysics, 5, 65-80. 
Matin, L., Matin, E., \& Pola, J. (1970). Visual perception of direction when voluntary saccades occur: II. Relation of visual direction of a fixation target extinguished before a saccade to a subsequent test flash presented before the saccade. Perception \& Psychophysics, 8 , 9-14.

Matin, L., \& Pearce, D. G. (1965). Visual perception of direction for stimuli flashed during voluntary saccadic eye movements. Science, 148, 1485-1488.

Matin, L., Pearce, D. G., Matin, E., \& Kibler, G. (1966). Visual perception of direction in the dark: Roles of local sign, eye movements, and ocular proprioception. Vision Research, 6, 453-469.

Matin, L., Picoult, E., Stevens, J. K., Edwards, M. W., Young, D., \& MACARThUR, R. (1982). Oculoparalytic illusion: Visual-field dependent spatial mislocalizations by humans partially paralyzed with curare. Science, 216, 198-201.

MAYs, L. E., \& SPARKs, D. L. (1980). Saccades are spatially, not retinocentrically, coded. Science, 208, 1163-1165.

Mclaughlin, S. C. (1967). Parametric adjustment in saccadic eye movements. Perception \& Psychophysics, 2, 359-362.

Mclaughuin, S. C., Kelly, M. J., Anderson, R. E., \& Wenz, T. G. (1968). Localization of a peripheral target during parametric adjustment of saccadic eye movements. Perception \& Psychophysics, 4, 45-48.

MILLER, J. M. (1980). Information used by the perceptual and oculomotor systems regarding the amplitude of saccadic and pursuit eye movements. Vision Research, 20, 59-68.

Mittelstaedt, $H$. (1958). The analysis of behavior in terms of control systems. In B. Schaffner (Ed.), Group processes, Transactions of the Fifth Conference. New York: Josiah Macy, Jr., Foundation.

ONo, H., \& NakAmizo, S. (1977). Saccadic eye movements during changes in fixation to stimuli at different distances. Vision Research, 17, 233-238.

O'Regan, J. K. (1984). Retinal versus extraretinal influences in flash localization during saccadic eye movements in the presence of a visible background. Perception \& Psychophysics, 36, 1-14.

PoLA, J. (1976). Voluntary saccades, eye position and perceived visual direction. In A. Monty \& J. W. Senders (Eds.), Eye movements and psychological processes. New York: Erlbaum.

Robinson, D. (1975). Oculomotor control signals. In G. Lennerstrand, P. Bach-y-Rita, C. C. Collins, A. Jampolsky, \& A. B. Scott (Eds.), Basic mechanisms of ocular motility and their clinical implications. New York: Pergamon Press.

Shebilske, W. L. (1976). Extraretinal information in corrective saccades and inflow vs. outflow theories of visual direction constancy. Vision Research, 16, 621-628.

Sherrington, C. S. (1918). Observations on the sensual role of the proprioceptive neural supply of the extrinsic ocular muscles. Brain, 41, 332-345.

SirBeCK, R. (1953). Wahrnehmungsformen bei experimentellen Augenmuskellahmungen. Deutsche Ophthalmologische Gesellschafi, 58, 24.

SirbeCK, R. (1954). Wahrnehmungsstorung und Storungswahmehmung bei Augenmuskellahmungen. von Gräfes Archiv für Ophthalmologie, 155, 26-34.

SIEBECK, R., \& FREY, R. (1953). Die Wirkungen muskeleschlaffender Mittel auf die Augenmuskeln. Anaesthesist, 2, 138-141.

SKAVENSKI, A. A. (1976). The nature and role of extraretinal eye-position information in visual localization. In A. Monty \& J. W. Senders (Eds.), Eye movements and psychological processes. New York: Erlbaum.

Skavenski, A. A., Steinman, R. M. (1970). Control of eye position in the dark. Vision Research, 10, 193-203.

SPERRY, R. W. (1950). Neural basis of the spontaneous optokinetic response produced by visual neural inversion. Journal of Comparative \& Physiological Psychology, 43, 482-489.

Stevens, J. K., Emerson, R. C., Gerstein, G. L., Kallos, T., NeuFELD, G. R., Nichols, C. W., \& Rosenquist, A. C. (1976). Paraly- sis of the awake human: Visual perceptions. Vision Research, 16, 93-98.

von Holst, E., \& Mittelstaedt, H. (1950). Das Reafferenzprinzip. Naturwissenshaften, 37, 464-476.

Weber, R. B., \& DARoff, R. B. (1972). Corrective movements following refixation saccades: Type and control system analysis. Vision Research, 12, 467-475.

WEST, R. (1932). Curare in man. Royal Society of Medicine, Proceedings, 25, 1107-1116.

WOODWORTH, R. S., \& SCHLOSBERG, H. (1954). Experimental psychology. New York: Holt, Rinehart, \& Winston.

Young, L. R., \& Stark, L. (1963a). A discrete model for eye tracking movements. Institute of Electrical and Electronic Engineers Transactions on Military Electronics, MIL-7, 113-115.

Young, L. R., \& STARK, L. (1963b). Variable feedback experiments testing a sample data model for eye tracking movements. Institute of Electrical and Electronic Engineers Transactions on Human Factors in Electronics, HFE-4, 38-51.

Zee, D. S., Optican, L. M., CooK, J. D., Robinson, D. A., \& King ENGEL, W. (1976). Slow saccades in spinocerebellar degeneration. Archives of Neurology, 33, 243-251.

\section{NOTES}

1. In an important, if obscure, paper he contributed to a symposium on group processes, Mittelstaedt (1958) used control-system techniques to reanalyze the functional schemata he and von Holst had advanced in their classic paper (von Holst \& Mittelstaedt, 1950). Hershberger (1976) extended this analysis to illustrate two points, both consistent with Mittelstaedt's analysis: (1) von Holst and Mittelstaedt's efference copy is not a sollwert (reference signal); neither afference nor reafference can be driven into correspondence with the efference copy by means of negative feedback. (2) Negative feedback can only drive efference into correspondence with a central command signal, which Mittelstaedt labels simply as " $C$." This sollwert, $C$, is neither afference nor efference, but, since it serves as the neural signal that afference "strives" to imitate, may be regarded as an afference copy in the archaic sense of the term "copy," meaning that which is to be imitated.

2. A light-emitting diode pulsed 120 times a second, a rate well in excess of the critical fusion frequency, works well, as does a simple nightlight: General Electric makes a $1 / 4-W$ neon nightlight that blinks at $120 \mathrm{~Hz}$. To keep the environment dark, it is necessary to mask off much of the nightlight with opaque tape. However, the dim illumination of the environment provided by the naked nightlight does not destroy the phenomenon.

3. The afference-copy hypothesis posits a discrete (abrupt) shift in retinal local signs that occurs sometime before or during its attendant saccade. The gradual shift apparent in Matin's data is assumed to reflect the continuously increasing cumulative probability that the discrete shift has occurred by the time indicated.

4. Although this procedure amounts to a graphic averaging of each subject's saccades, only one of the three functions appears to be saccadic (J.P.'s). The asymptotes for the other two functions are too belated for those functions to be exclusively saccadic. This is particularly true for E.M., whose voluntary saccades appear to have been accompanied by rapid pursuit eye movements that persisted after her saccades. In any event, something was confounded with the simple, $2^{\circ}$ voluntary saccade she ostensibly was making, thereby rendering her psychophysical judgments difficult, if not impossible, to interpret. L.M.'s eye-position data appear similarly contaminated, but to a lesser degree. Because only J.P.'s eye-position data appear to be free of this contamination, only his psychophysical judgments can be taken at their face value.

(Manuscript received November 27, 1985; revision accepted for publication October 8,1986 .) 